\title{
Transmisión interepidémica del dengue en la ciudad de Colima, México
}

\author{
Francisco Espinoza-Gómez, Dr en C, ${ }^{(1)}$ Carlos Moisés Hernández-Suárez, PhD, ${ }^{(2)}$ Ruth Rendón-Ramírez, Q F, ${ }^{(3)}$ \\ Mayra Lizet C arrillo-Alvarez, MP,(1) Juan Carlos Flores-González, MP.(1)
}

\begin{abstract}
Espinoza-Gómez F, Hernández-Suárez CM, Rendón-Ramírez R, Carrillo-Alvarez ML, Flores-González JC. Transmisión interepidémica del dengue en la ciudad de Colima, México. Salud Publica Mex 2003;45:365-370. El texto completo en inglés de este artículo está disponible en: http://www.insp.mx/salud/index.html
\end{abstract}

\section{Resumen}

Objetivo. Determinar la presencia de dengue interepidémico en una co munidad infestada por Aedes aegypti, y analizar sus características epidemiológicas. Material y métodos. Entre 2001 y 2002 se realizó un estudio probabilístico longitudinal en 245 habitantes de la ciudad de Colima, México. En cada caso se registraron: edad, sexo, nivel socioeconómico, cuadro clínico sugestivo de dengue, y se buscó la presencia de lgG e IgM antidengue por inmunocromatografía rápida y por ELISA, en un seguimiento de siete meses. Los datos se analizaron mediante tablas de contingencia y regresión de Poisson univariada. Resultados. D oce individuos resultaron con infección reciente (incidencia de 1.77\%; IC 95\%: 0.9-3.1\%), ocho de ellos refirieron cuadro clínico reciente $\left(\chi^{2}=19.6 ; p=0.0001, \mathrm{RM}: 19.6\right)$. La regresión de Poisson no reveló correlación de la infección reciente con edad, sexo, ni con nivel socioeconómico. Conclusiones En comunidades infestadas por A aegypti, co mo Colima, pueden ocurrir infecciones continuas por dengue sin epidemia aparente. Dichas infecciones no parecen asociarse con la edad, el sexo, ni con el nivel socioeconómico, pero sí con el cuadro clínico, el cual podría considerarse como indicador precoz de posible transmisión interepidémica. El texto completo en inglés de este artículo está disponible en: http://www.insp.mx/salud/index.html

Palabras clave: dengue; IG M; Colima; México

\author{
Espinoza-Gómez F, Hernández-Suárez CM, \\ Rendón-Ramírez R, Carrillo-Alvarez ML, Flores-González JC. \\ Interepidemic transmission of dengue \\ in Colima City, Mexico. \\ Salud Publica Mex 2003;45:365-370. \\ The English version of this paper \\ is available at: http://www.insp. mx/salud/index.html
}

\begin{abstract}
A bstract
Objective. To determine the occurrence of interepidemic dengue in a community infested with Aedes aegypti and to analyze its epidemiologic characteristics. Material and MethodsA longitudinal probabilistic study was car ried out in Colima City between 2001 and 2002. The sample population consisted of 245 subjects.T he following were recorded for each subject: sex, age, socio-economic level and dengue fever symptoms, testing for serum IgG and IgM antidengue, using quick immunochromato graphy as well as ELISA tests, in a seven-month follow-up period. Results. Twelve individuals showed recent dengue infection (incidence: 1.77\%; 95\%: confidence interval 0.9-3.1\%). Of these, eight had recent clinical symptoms $\left(\chi^{2}=19.6 ; p<0.0001,0\right.$ dss ratio 19.6). Poisson regression analysis showed no evidence of correlation between recent infection and sex, age, or socioeconomic level. Conclusions Communities infested with A. aegypti, like Colima, may continually exhibit dengue infection with no apparent epidemic. Such infections are seemingly not associated with age, sex, or socioeconomic level, but with clinical symptoms. This situation could be considered an early indicator of interepidemic transmission. The English version of this paper is available at:http://www.insp.mx/ salud/index.html
\end{abstract}

Key words: dengue; IG M; Colima; Mexico

Este programa fue financiado por el Sistema de Investigación Regional Simorelos (financiamiento N 0. 19990302001) y por el Fondo para la Investigación "Ramón Alvarez Buylla de Aldana".

(1) Facultad de Medicina de la Universidad de Colima. Colima, Colima, México.

(2) Facultad de Ciencias de la Universidad de Colima. Colima, Colima, México.

(3) Facultad de Ciencias Q uímicas de la Universidad de Colima. Colima, Colima, México.

Fecha de recibido: 15 de noviembre de 2002 - Fecha de aceptado: 19 de mayo de 2003 Solicitud de sobretiros: Dr. Francisco Espinoza Gómez. Facultad de Medicina de la Universidad de Colima. Avenida Universidad 333, colonia Las Víboras, 28040, Colima, Colima, México. Correo electrónico: fespin@ cgic.ucol.mx 
A ctualmente, las epidemias por dengue son uno de los problemas de salud pública más relevantes en la mayor parte de las comunidades urbanas de los países tropicales. ${ }^{1}$ Tradicionalmente se piensa que dichas epidemias inician cuando una persona infectada por algún serotipo nuevo ingresa en una comunidad donde hay una gran proporción de individuos susceptibles, iniciándose así la epidemia, que se expande en tiempo y espacio hasta que el nivel de infectados e inmunizados inhibe su transmisión, con lo que sobreviene una declinación progresiva, hasta su extinción. ${ }^{2,3}$

Sin embargo, en zonas tropicales urbanas persistentemente infestadas por mosquitos vectores del dengue, en particular por el Aedes aegypti (L), es fácil suponer que las epidemias aparezcan más bien a partir de reservorios naturales dentro de la propia comunidad, como consecuencia de cambios bruscos en la dinámica de la población humana, de vectores, o de virus, $^{2,4,5}$ independientemente de la importación de nuevas infecciones desde otra localidad.

De esta manera, se podría señalar que existen lugares donde el dengue ha adquirido un equilibrio endémico, con una perpetua transmisión de baja intensidad, la cual puede pasar desapercibida para los sistemas de vigilancia epidemiológica tradicionales, debido a que un alto porcentaje de individuos infectados cursan asintomáticos, o bien, porque su sintomatología es tan leve que no amerita la búsqueda de consulta médica, aunque de todas formas participan en la cadena de transmisión. ${ }^{6,7}$

En tales circunstancias, la estimación de la incidencia real del dengue se debe fundamentar en estudios activos probabilísticos, con seguimiento de cohortes ${ }^{8}$ más que en la captación pasiva de casos clínicos que acuden a consulta, como habitualmente se lleva a cabo en los programas de vigilancia epidemiológica. A pesar de la extensa literatura sobre la epidemiología del dengue son muy escasos los estudios orientados a la determinación de su incidencia en forma prospectiva debidamente aleatorizados y, en los pocos existentes, no se hace análisis de las características demográficas o clínicas que permitan la identificación de grupos poblacionales con mayor riesgo de infección, ${ }^{9,10}$ motivo por el que hasta ahora los factores de riesgo reconocidos, como el sexo femenino, el nivel socioeconómico bajo y la etapa de adulto joven, se han basado en encuestas pasivas y retrospectivas. ${ }^{2,11}$

Por otro lado, en las zonas endémicas es imprescindible definir qué tan confiable es la sintomatología sugestiva de dengue clínico cuando no hay evidencias de epidemia manifiesta, con el fin de validar la estrategia de vigilancia epidemiológica basada en la participación de la comunidad, que algunos autores han denominado "alerta de fiebre", 9,12 para lo cual se hace necesaria una determinación confiable de anticuerpos específicos de infección reciente, como son las $\operatorname{IgM}$ antidengue en los casos sospechosos referidos por la propia comunidad, y no solamente en aquellos captados por los servicios médicos.

La ciudad de Colima, México, es una zona permanentemente infestada por Aedes aegypti, ${ }^{13}$ con brotes epidémicos de dengue, el más reciente en 1997, cuando se alcanzó una cifra de 4851 casos de dengue clásico y 59 de dengue hemorrágico. ${ }^{14}$ Además, en la ciudad se ha documentado la circulación de al menos dos serotipos ${ }_{1}^{15}$ datos que catalogarían a ésta como una zona endémica, sin embargo, en vista de que durante el año 2000 solamente se registraron 15 casos nuevos, mientras que en 2001 ninguno, ${ }^{16}$ podría suponerse que la transmisión del dengue en Colima se debe más bien a la importación de casos en forma episódica. Con el propósito de verificar la existencia de transmisión interepidémica en la ciudad de Colima se llevó a cabo la presente encuesta seroepidemiológica probabilística, al tiempo que se exploró la asociación de dicha transmisión con ciertas variables epidemiológicas y con la presencia de cuadro clínico.

\section{Material y métodos}

El estudio consistió en un muestreo probabilístico que se llevó a cabo en habitantes de la ciudad de Colima, Colima, localizada en la costa del Pacífico mexicano $\left(19^{\circ}, 15^{\prime} \mathrm{LN}, 103^{\circ}, 43^{\prime} \mathrm{LO}\right)$, a 490 metros sobre el nivel del mar, con un clima semicálido y semiseco $\left(\mathrm{AWC}_{1}\right.$ de Köeppen), con una temperatura media anual de $23.5^{\circ} \mathrm{C}$ y una precipitación pluvial anual de $900 \mathrm{~mm}$. La zona urbana cuenta con una población de 182000 habitantes. ${ }^{17}$

Sobre el plano de la ciudad se trazó una cuadrícula de aproximadamente una hectárea por cada cuadro. Los cuadros se numeraron de manera secuencial y, enseguida, se escogieron 24 de ellos por medio de sorteo. Dentro de cada uno se seleccionaron, también de manera aleatoria, entre 10 y 20 personas, según la densidad poblacional estimada para las zonas en cuestión, de acuerdo con los datos demográficos de las áreas geográficas básicas en Colima, ${ }_{17}$ procurando incluir una proporción equilibrada de hombres, mujeres, niños y adultos.

El muestreo se realizó en dos etapas, una basal, durante la época calurosa y de lluvias (entre julio y septiembre de 2001), cuando tradicionalmente se reporta el mayor número de $\operatorname{casos}^{14} \mathrm{y}$ la segunda, en la temporada fría y seca (diciembre de 2001 a febrero de 2002), en la cual se hizo el seguimiento de una cohorte 
de las personas inicialmente encuestadas. El tamaño de la muestra originalmente planeado fue de 385 personas, suponiendo una prevalencia nacional estimada de $16 \%,{ }^{18}$ con una precisión de $4 \%$. A cada persona seleccionada se le solicitó su participación por medio de consentimiento escrito, previa explicación del procedimiento.

En cada caso se aplicó una encuesta en la que se consignaron los datos referentes a edad, sexo, y nivel socioeconómico, determinado en forma ordinal de acuerdo con la escala propuesta por Suadicani. ${ }^{19}$ Asimismo, se interrogó sobre si la persona había presentado cuadro sugestivo de dengue o "trancazo", como se conoce comúnmente a la enfermedad en Colima (fiebre, acompañada de dolores óseos y dolor retrocular, sin datos de gripe o de enfermedad diarreica), durante los dos meses previos a la encuesta o en el periodo de seguimiento.

Al momento de la encuesta se tomaron $5 \mathrm{ml}$ de sangre venosa, y se separó el suero para congelación a $-20{ }^{\circ} \mathrm{C}$. De esta manera se colectaron 433 muestras de suero al inicio del estudio (previendo una pérdida de $10 \%$ ), mientras que en el segundo muestreo se rescataron 245 de las muestras iniciales.

Los sueros fueron procesados con la técnica de inmunocromatografía rápida (ICR) (PanBio test $®)$, cuyo procedimiento ha sido detallado en publicaciones previas, ${ }^{20}$ para determinación de IgG e IgM antidengue. Sólo se consideraron positivos aquellos sueros con coloración francamente aparente. En una submuestra de 127 sueros se practicó prueba confirmatoria para IgM e IgG antidengue por medio de la prueba ELISA (EIA Light Diagnostics, Chemicon, Int, Inc. Temecula CA. USA).

Los sueros positivos a IgG durante el primer muestreo fueron considerados como infecciones previas, con lo que se estimó la prevalencia de punto para infecciones por dengue, independientemente del serotipo y del tiempo en que éstas hubieran ocurrido. Se consideraron como infecciones recientes o "casos nuevos" aquellos sueros positivos a IgM en cualquiera de los dos muestreos (asumiendo que IgM permanece positiva entre 7 a 90 días después de la infección), ${ }^{20}$ así como los que mostraron seroconversión a IgG en el segundo muestreo.

La asociación entre la infección reciente con la presencia de cuadro clínico se analizó con tablas de doble entrada y prueba exacta de Fisher, en tanto que la correlación entre dicha infección reciente, con las características demográficas, expresadas como variables categóricas múltiples, se exploró por medio de regresión de Poisson univariada, utilizando el programa PEPI v 4.0. ${ }^{21}$ Para la edad se analizaron siete niveles, de acuerdo con décadas de la vida; para nivel socioeconómico se emplearon tres categorías (bajo, medio y alto, según la clasificación de Suadicani) ${ }_{1}^{19}$ mientras que el sexo se analizó en dos categorías. Para cada nivel se calculó la tasa de proporción (rate ratio) y su valor $p$ de Wald respecto al nivel de referencia.

\section{Resultados}

De las 433 muestras de sangre obtenidas durante el primer muestreo, 142 resultaron positivas a IgG y cuatro a IgM, mientras que en el segundo, realizado en promedio 7.2 meses después, se rescataron 245 sueros, habiéndose perdido 188 de ellos (43.4\%). La causa de dicha pérdida se atribuyó a cambio de domicilio en 125 casos, renuencia para seguir colaborando con el estudio en 60 y a problemas médicos en tres personas. De los 245 sueros que se tomaron en la segunda fase, 50 fueron positivos a IgG; dos de ellos en sujetos previamente negativos, mientras que seis resultaron positivos a IgM, lo que se interpretó como aparición de cuatro casos nuevos durante el primer muestreo y ocho durante el seguimiento. En las 127 muestras analizadas con ELISA para IgM e IgG se encontró una sensibilidad de $99.8 \%$ (IC 95\%: 92-99.9\%) y especificidad de 98\% (IC 95\%: 91.5-99.2\%) para IgM, mientras que para IgG la sensibilidad resultó de 75\% (IC 95\%: 65- 83\%) y la especificidad de 100\% (IC 86.3- 99.7\%).

En el cuadro I se ilustran los resultados serológicos obtenidos por ICR en ambos muestreos, con el número total de casos nuevos y su correlación con las variables demográficas; allí mismo se muestran las tasas de prevalencia basal y de incidencia durante el periodo estudiado, y se destaca que ninguno de los casos nuevos salió de la ciudad durante dicho lapso.

Como puede apreciarse en el mismo cuadro, la correlación entre la incidencia de casos nuevos y las variables demográficas analizadas por regresión de Poisson univariada no mostraron asociación significativa con ninguna de ellas, a juzgarse por los valores de la tasa de proporción y la $p$ de Wald.

En relación con la distribución espacial y temporal de los casos nuevos, cuatro de ellos mostraron coincidencia espacial (dentro del bloque de muestreo, pero no en la misma familia), mientras que, de acuerdo con la aparición de IgM, hubo una aparente coincidencia temporal de cuatro en el primer muestreo y seis en el segundo; sin embargo, al analizar la probabilidad de conglomerados, solamente en dos sujetos hubo coincidencia espacial y temporal, por lo que el valor esperado para conglomerados con la prueba de Knox no alcanzó un nivel estadístico significativo (2.66, $p=0.74)$. 


\section{Cuadro I \\ Casos nuevos de infección por dengue y su correlación con las variables epidemiológicas exploradas. Colima, Colima, México, 2001-2002}

\begin{tabular}{|c|c|c|c|c|c|c|c|c|c|}
\hline \multirow[b]{2}{*}{ Variable } & \multicolumn{3}{|c|}{ Primer muestreo } & \multicolumn{3}{|c|}{ Segundo muestreo } & \multirow[b]{2}{*}{ Caso nuevo } & \multicolumn{2}{|c|}{ Regresión Poisson } \\
\hline & $n$ & $\lg G(+)$ & $\lg M(+)$ & $n$ & $\lg G(t)$ & $\lg M(+)$ & & Tasa de proporción & pWald \\
\hline \multicolumn{10}{|l|}{ Sexo } \\
\hline Femenino & 305 & 104 & 4 & 173 & 37 & 3 & 8 & - & - \\
\hline Masculino & 128 & $38^{*}$ & 0 & 72 & 13 & 3 & 4 & 0.84 & 0.77 \\
\hline
\end{tabular}

Edad

\begin{tabular}{lrlllrlrll}
$0-9$ & 49 & 13 & 0 & 28 & 4 & 0 & 1 & - & - \\
\hline $10-19$ & 107 & 36 & 1 & 68 & 15 & 2 & 4 & 1.56 & 0.71 \\
\hline $20-29$ & 69 & 18 & 0 & 35 & 5 & 1 & 1 & 0.61 & 0.72 \\
\hline $30-39$ & 67 & 23 & 0 & 38 & 7 & 1 & 1 & 0.45 & 0.57 \\
\hline $40-49$ & 73 & 25 & 1 & 37 & 8 & 1 & 2 & 0.45 & 0.57 \\
\hline $50-59$ & 37 & 17 & 1 & 23 & 7 & 1 & 2 & 0.86 & 0.9 \\
\hline$\geq 60$ & 31 & 10 & 1 & 16 & 4 & 0 & 1 & 1.07 & 0.94
\end{tabular}

$\mathrm{N}$ ivel socioeconómico

\begin{tabular}{lrrrrrrrrl} 
Bajo & 239 & 78 & 2 & 152 & 26 & 5 & 8 & - & - \\
\hline Medio & 135 & 48 & 2 & 66 & 18 & 1 & 3 & 1.28 & 0.83 \\
\hline Alto & 59 & $16^{\ddagger}$ & 0 & 27 & 6 & 0 & 1 & 1.76 & 0.6 \\
\hline Totales & 433 & $142^{\S}$ & 4 & 245 & $50^{\#}$ & 6 & $12^{\S}$ & &
\end{tabular}

* Prevalencia en sexo femenino vs masculino $\chi^{2}=0.6, p=0.43,0 R=1.22$ (IC 95\%: 0.76-1.96)

₹ $\mathrm{N}$ ivel socioeconómico bajo vs alto: $\chi^{2}=0.43, p=0.5,0 \mathrm{R}=1.3$ (IC 95\%: 0.66-2.58)

§ Prevalencia de punto basal: $32.8 \%$ (IC 95\%: 28.36-37.2)

\# De los lgG positivos en $2^{\circ}$ muestreo, dos habían sido negativos y se consideran casos nuevos

\& Incidencia en 7.2 meses: $1.77 \%$ (IC 95\%: 0.9-3.1\%)

En el cuadro II se ilustra la asociación entre caso nuevo, de acuerdo con el criterio arriba señalado y el cuadro clínico sugestivo de dengue.

\section{Discusión}

La prevalencia de punto para infecciones por dengue de $32.8 \%$ encontrada en este estudio puede estar subestimando la prevalencia real si tomamos en cuenta la baja sensibilidad de la prueba ICR para IgG, fenómeno recientemente reportado por otros autores, ${ }^{22}$ a pesar de lo cual se obtuvieron valores sensiblemente mayores a los de la media nacional ${ }^{19}$ y semejantes a los encontrados en áreas endémicas, ${ }^{9}$ lo que hace suponer la presencia de epidemias repetidas, o de una transmisión endémica continua en la zona.

El estudio permitió la identificación de infecciones recientes por dengue aun en ausencia de brote epidémico en la zona urbana de Colima, con base en la presencia de anticuerpos $\operatorname{IgM}$ o $\operatorname{IgG}$ de reciente aparición; dichas infecciones son autóctonas, ya que ningu-

\section{Cuadro II \\ Asociación ENTRE CUADRo CLÍNICO E INFECCIÓN reciente por dengue (IgM). Colima, Colima, MÉxıCo, 2001-2002}

$$
\begin{array}{lr}
\text { Infección reciente por dengue } \\
\hline \text { Positiva }
\end{array}
$$

Cuadro clínico reciente

\begin{tabular}{llr} 
Positivo & $9 *$ & 62 \\
\hline N egativo & 3 & 614
\end{tabular}

$* \chi^{2}=29.7, \mathrm{DF}=2, \mathrm{p}=0.00001, \mathrm{RM}=19.6$

₹ Síntomas compatibles con dengue durante los dos meses previos a la encuesta. Se contabilizan datos completos de ambas encuestas ( 428 basales y 248 posteriores)

na de las personas salió de la ciudad en el periodo de estudio. En este sentido, podría argumentarse que la prueba de ICR sobrestima la presencia de infecciones por dengue debido a una baja especificidad, ya que en 
zonas donde existe fiebre amarilla o encefalitis japonesa pueden presentarse casos falso positivos a IgM antidengue, ${ }^{20}$ sin embargo, en Colima no se ha detectado la primera por más de 60 años y no es zona donde se transmita la segunda; además, la confirmación con ELISA revela que la especificidad es muy aceptable $(98 \%)$, tal como originalmente fue encontrado por los autores que proponen la prueba, mientras que la sensibilidad respecto a ELISA mostró ser también muy confiable, por lo cual el cálculo de incidencia se hizo en relación con la prueba de ICR, tanto para IgM en los dos muestreos, como para la aparición de IgG en sujetos previamente seronegativos.

El tamaño de la muestra no fue el inicialmente planeado para la cohorte, debido principalmente a la intensa movilidad de las personas, y pudiera considerarse que dicho número no es suficientemente representativo de la población en estudio, sin embargo, el intervalo de confianza de $95 \%$ (entre 0.9 a $3.1 \%$ ), de todas formas permite suponer, con un margen razonable de certeza, que en la ciudad de Colima existe una transmisión endémica continua que puede pasar desapercibida para los sistemas de vigilancia epidemiológica tradicionales, debido a que dichos sistemas habitualmente se basan en los enfermos que acuden a recibir consulta médica,, 78 mientras que, como ya se señaló, puede existir una transmisión "silenciosa" en la que los individuos infectados no presentan manifestación clínica alguna, ${ }^{6,23} \mathrm{o}$ bien, porque los médicos normalmente no diagnostican dengue cuando no existen brotes epidémicos francos.

Generalmente se considera que las epidemias por dengue se originan a partir de la importación de serotipos nuevos acarreados por personas infectadas, provenientes de zonas afectadas, ${ }^{2,3}$ sin embargo, los presentes hallazgos sugieren que más bien en zonas permanentemente infestadas por Aedes aegypti, como se ha demostrado en el caso de la ciudad de Colima, ${ }^{13}$ el dengue mantiene un equilibrio endémico a través de una transmisión continua, la cual sirve de reservorio para eventuales brotes epidémicos que se pueden presentar cuando las poblaciones del vector alcanzan niveles críticamente elevados. ${ }^{4,24}$ Considerando lo anterior, la alta incidencia de infecciones recientes por dengue en Colima sugiere el inicio de una transmisión en ascenso, que pudiera traducirse en un riesgo inminente de epidemia en la zona.

En vista de que la probabilidad de infecciones nuevas por dengue es menor a 5\% se utilizó análisis probabilístico con regresión de Poisson para explorar la correlación de dicho evento con edad, sexo y nivel socioeconómico. A juzgar por los resultados de dicho análisis, las infecciones nuevas ocurren en la comuni- dad sin relación con ninguna de dichas variables, lo cual permite suponer que existen otros factores de mayor peso en el riesgo de transmisión, como podrían ser los parámetros entomológicos en las zonas donde habitan las personas estudiadas.

Por otro lado, la presencia de manifestaciones clínicas referidas por la comunidad como dengue o "trancazo" mostró una fuerte asociación con la aparición de anticuerpos antidengue, lo cual podría apoyar la propuesta de algunos autores en el sentido de no desestimar la llamada "alerta de fiebre", como una forma de vigilancia epidemiológica basada en la comunidad. ${ }^{8,12}$

A manera de conclusión, en gran parte de las zonas infestadas por el vector pueden existir focos de transmisión endémica del dengue, que sirven de reservorio para posteriores epidemias. Se recomienda que todo sistema de vigilancia epidemiológica en tales zonas debería incluir seguimientos de cohorte debidamente aleatorizadas, con el fin de estimar su incidencia real y que los centros de atención médica estén siempre alerta ante la referencia de cuadro clínico sugestivo de dengue por parte de las personas de la comunidad, como un posible indicador temprano de transmisión interepidémica.

\section{A gradecimientos}

A los profesores de la Facultad de Medicina de la Universidad de Colima: al M en C Arcadio Maldonado Rodríguez y al doctor Rafael Coll Cárdenas; a los estudiantes de la misma escuela: Tania Uribe, Paloma Liñán, Julio César Barragán Anaya, Landy Sahagún y Dianella Portillo, por su valiosa colaboración en el trabajo de campo y captura de datos, así como al químico Baltasar Briceño G del Departamento de virología, del Instituto Nacional de Referencia Epidemiológica, por su apoyo en los estudios serológicos.

\section{Referencias}

1. Gubler DJ. D engue and dengue hemorrhagic fever. Clin Microbiol Rev 1998;11:480-496.

2. Kuno $\mathrm{G}$. Review of the factors modulating dengue transmission. Epidemiol Rev 1995;17:321-335.

3.Teixeira MG , Costa MC, Barreto ML, Barreto FR. Epidemiology of dengue in Salvador-Bahia, 1995-1999. Rev Soc Bras Med Trop 2001;34:269-274.

4. Woodring JL, Higgs S, Beaty B. N atural cycles of vector- borne pathogens, En: Beaty J, Marquadt W, Ed.The biology of disease vectors. (CO ):University Press of Colorado, 1996.

5.W ittke V, Robb T, Thu H, N isalak A, N immannitya S, Kalayanrooj S et al. Extinction and rapid emergence of strains of dengue 3 virus during an interepidemic period.Virology 2002;30:148-156. 
6. Chen W J, Chen SL, Chien LJ, Chen CC, King CC, Harn MR et al. Silent transmission of the dengue virus in southern Taiwan. Am J Trop Med Hyg 1996;55:12-16.

7. Rigau-Pérez JG, A yala-López A,Vorndam AV, Clark G G. D engue activity in Puerto Rico during an interepidemic period (1995-1997). Am J Trop Med Hyg 2001;64(1-2):75-78.

8. G ubler DJ.Active surveillance for dengue and dengue hemorrhagic fever. Bol 0 ficina Sanit Panam 1989;107:22-30.

9. Graham RR, Juffrie M, Tan R, Hayes CG , Laksono I, Ma'roef C et al. A prospective seroepidemiologic study on dengue in children four to nine years of age in Yogyakarta, Indonesia I, studies in 1995-1996. Am J Trop Med Hyg 1999;61:412-419.

10.Vasconcelos PF, Lima JW, da Rosa AP, T imbo MJ, da Rosa ES, Lima HR et al. Dengue epidemic in Fortaleza, C eara: Randomized seroepidemiologic survey. Rev Saude Publica 1998; 32:447-454. 11. Koopman J, Prevots D R, Vaca MA, Gómez-D antés H. D eterminants and predictors of dengue infection in Mexico.Am J Epidemiol 1999;133: 1168-1178.

12. Condon R, Taleo G, Stewart T, Sweeney T, Kiedrzynski T. D engue surveillance in the Pacific Islands. Pac Health Dialog 2000;7:122-126. 13. Espinoza-G ómez F, Hernández-Suárez CM, Coll-Cárdenas R. Factores que modifican los índices larvarios de Aedes aegypti en Colima, México. Rev Panam Salud Publica 2001;10:6-12.

14. Instituto Mexicano del Seguro Social, Boletín estadístico anual de mortalidad 1997. México, D F: Instituto Mexicano del Seguro Social, 1998.

15. Loroño-Pino MA, Cropp C B, Farfán JA,Vorndam AV, RodríguezAngulo EM, Rosado-Paredes EP et al. Common occurrence of concurrent infections by multiple dengue virus serotypes. Am J Trop Med Hyg 1999 N ov;61(5):725-730.
16. Secretaría de Salud. Sistema único de información y vigilancia 2001. Casos por entidad federativa de enfermedades transmisibles por vector. Vigilancia Epidemio lógica. Semana 52, 2001, cuadro 7.1. Secretaría de Salud, México, DF: 2001

17. Instituto $\mathrm{N}$ acional de Estadística, Geografía e Informática. Sistema para la consulta de información censal. 2000. Colima, México: IN EG I, 2002

18. Gómez-D antés H, Montesano-C astellanos R, López-Moreno S, Tapia-C onyer R. D engue en México. Situación epidemiológica actual. Gac Med Mex 1995; 131:237-240.

19. Suadicani P, Hein HO, Gyntelberg F. Socioeconomic status and ischaemic heart disease mortality in middle-aged men: Importance of the duration of follow-up.The Copenhagen Male Study. Int J Epidemiol 2001; 30: 248-255

20. Lam SK, D evine PL. Evaluation of capture ELISA and rapid immunochromatographic test for the determination of IgM and IgG antibodies produced during dengue infection. C lin Diagn Virol. 1998; 10:75-81.

21. A bramson JH, Gahlinger PM. Computer programs for epidemiologic analyses: PEPI v. 4.0. Salt Lake City (UT): Sagebrush Press, 2001.

22. Groen J, Koraka P, Velzing J, C opra C, 0 sterhaus AD. Evaluation of six immunoassays for detection of dengue virus-specific immunoglobulin $M$ and $G$ antibodies. Clin Diagn Lab Immunol 2000;7:867-871.

23. Halstead SB, Streit TG, Lafontant JG, Putvatana R, Russell K, Sun W et al. Haiti:A bsence of dengue hemorrhagic fever despite hyperendemic dengue virus transmission. Am J Trop Med Hyg 2001;65:180-183.

24. Hay SI, Myers MF, Burke DS, Vaughn DW, Endy T, Ananda N et al. Etiology of interepidemic periods of mosquito-borne disease. Proc $\mathrm{N}$ atl Acad Sci U SA 2000;97:9335-9339. 